\title{
Signals features extraction in radioisotope liquid-gas flow measurements using wavelet analysis
}

\author{
Robert Hanus ${ }^{1, *}$, Marcin Zych ${ }^{2}$, Barbara Wilk ${ }^{1}$, Marek Jaszczur ${ }^{3}$, and Dariusz Świsulski ${ }^{4}$ \\ ${ }^{1}$ Rzeszów University of Technology, Faculty of Electrical and Computer Engineering, 35-959 Rzeszów, Poland \\ ${ }^{2}$ AGH University of Science and Technology, Faculty of Geology, Geophysics and Environmental Protection, \\ 30-059 Kraków, Poland \\ ${ }^{3}$ AGH University Science and Technology, Faculty of Energy and Fuels, 30-059 Kraków, Poland \\ ${ }^{4}$ Gdańsk University of Technology, Faculty of Electrical and Control Engineering, 80-233 Gdańsk, Poland
}

\begin{abstract}
Knowledge of the structure of a flow is significant for the proper conduct of a number of industrial processes. In this case, a description of a two-phase flow regimes is possible by use of the timeseries analysis in time, frequency and state-space domain. In this article the Discrete Wavelet Transform (DWT) is applied for analysis of signals obtained for water-air flow using gamma ray absorption. The presented method was illustrated by use data collected in experiments carried out on the laboratory hydraulic installation with a horizontal pipe, equipped with two Am-241 radioactive sources and scintillation probes with $\mathrm{NaI}(\mathrm{Tl})$ crystals. Signals obtained from detectors for slug, plug, bubble, and transitional plug - bubble flows were considered in this work. The recorded raw signals were analyzed and wavelet energy was extracted using multiresolution analysis. It was found that energies of wavelet approximation at 1-5 levels are useful to recognize the structure of the flow.
\end{abstract}

\section{Introduction}

Knowledge of a two-phase flow regime is significant for the proper conduct of a number of industrial processes. Flow regime identification inspires many studies, Recent works describes a use for this purpose machine learning methods as e.g. artificial neural networks [112]. Generally, computational intelligence methods utilize various features of signals in the time, frequency and state-space domain. Features of signals in the frequency domain may be determined using the Fourier Transform, Short-Time Fourier Transform, wavelet transform and other methods [11,13,14].

In this work, wavelet transform is applied to signals obtained in two-phase liquid-gas flow measurements using gamma-ray absorption. The data were collected in experiments carried out on a laboratory hydraulic installation with a horizontal plexiglas pipe. Signals obtained for four structures of air-water flow as slug, plug, bubble, and transitional plug - bubble one were considered. The recorded raw signals were analyzed and energies of their wavelet decomposition were extracted using multiresolution analysis.

\section{Gamma-absorption method and laboratory set-up}

The gamma absorption technique is based on the exponential decay of a gamma radiation beam in function of composition and geometry of the absorbent
[9]. The changes of the intensity of radiation are detected by the scintillation probes and converted into electrical impulses.

The typical gamma-absorption set for two-phase liquid-gas flow measurement is presented in Fig. 1.

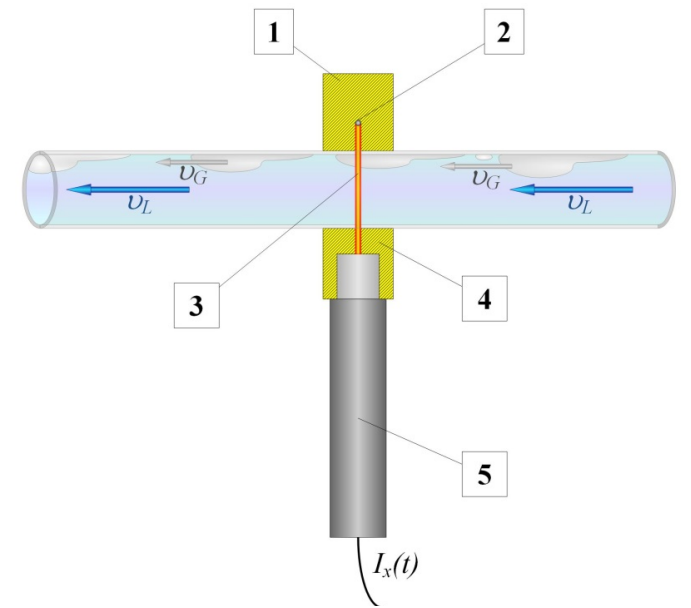

Fig. 1. The principle of gamma-absorption method: 1 collimator, 2 - gamma radioactive source, 3 - gamma ray, 4 collimator of detector, 5 - scintillation detector, $v_{G}$ - velocity of gas, $v_{L}-$ velocity of liquid [15].

The sealed radioactive source (2) emits gamma radiation beam (3) shaped by a collimator (1). Photons pass through the pipe and two-phase flows mixture. The scintillation detector (5) with a collimator (4) is placed on the other in referent to the source side of the pipe. In

Corresponding author: rohan@prz.edu.pl 
the presented experiment linear Am-241 source with an activity of $100 \mathrm{mCi}$ and probe with $\mathrm{NaI}(\mathrm{Tl})$ scintillation detector was used. At the output of the scintillation probe, count signal $I_{x}(t)$ is registered [15].

The gamma-absorption equipment described above was applied in the experimental measurement set-up, performed in Industrial Radiometry Laboratory of the AGH University of Science and Technology in Krakow, Poland. Measurement data were collected during experiments carried out on a laboratory hydraulic installation with a horizontal plexiglas pipe of $4.5 \mathrm{~m}$ length and the inner diameter of $30 \mathrm{~mm}$. A detailed description of experimental set-up can be found in [15, 16]. Figure 2 shows the general view of the measurement section of the installation.

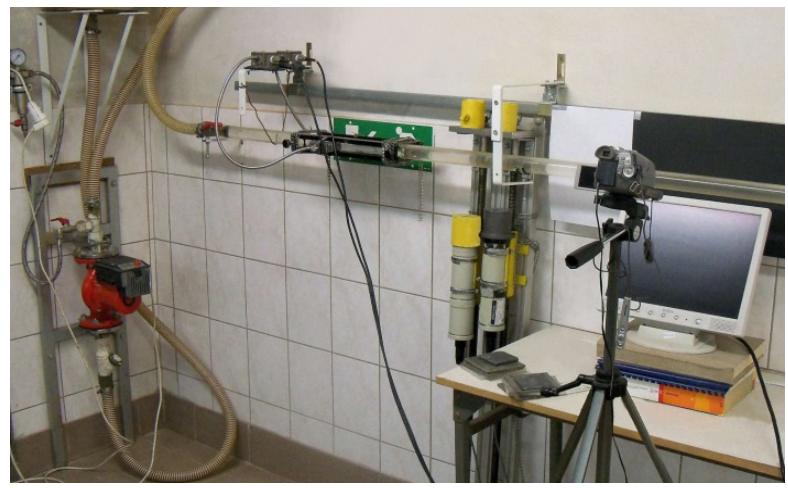

Fig. 2. General view of the experimental set-up.

Examples of the analyzed flow structures: slug (experiment LIW5), plug (LIW4), transitional plug bubble (LIW2) and bubble (LIW1) are presented in Fig. 3.

a)

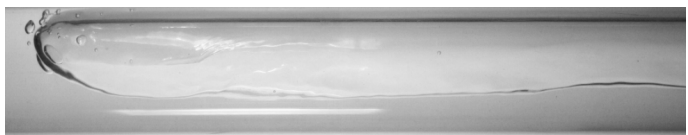

b)

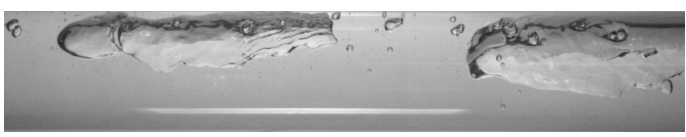

c)

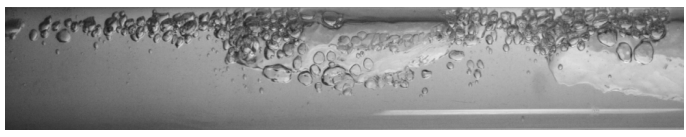

d)

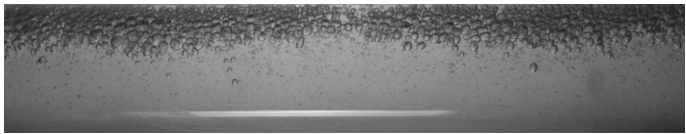

Fig. 3. Examples of the analysed flow structures: a) slug flow (LIW5), b) plug flow (LIW4), c) transitional plug - bubble flow (LIW2), d) bubble flow (LIW1).

The data acquisition system includes a 8-channel counter HSC 8000 connected to work station using a USB port. Voltage pulses $I_{x}(t)$ are counted within the sampling time $\Delta t=1 \mathrm{~ms}$ and create discrete signals $x(n)$. Exemplary time records of signals (after centering) obtained in the mentioned above experiments are presented in Fig. 4. a)

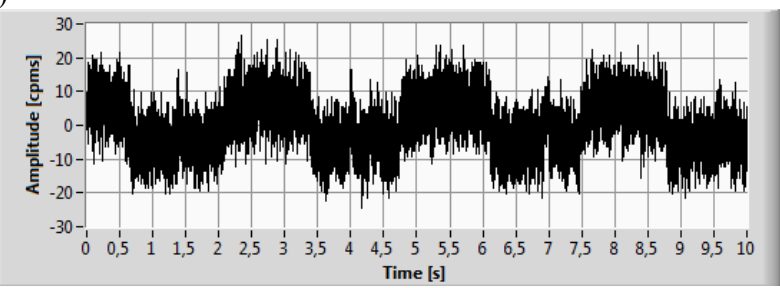

b)

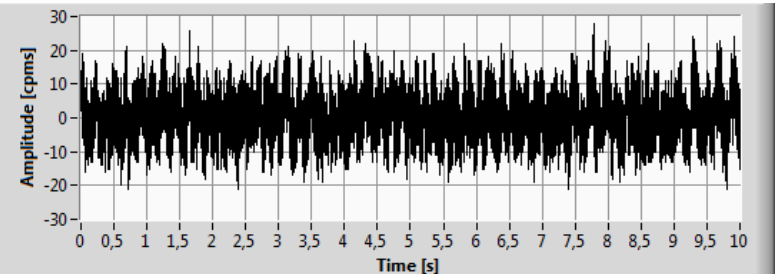

c)

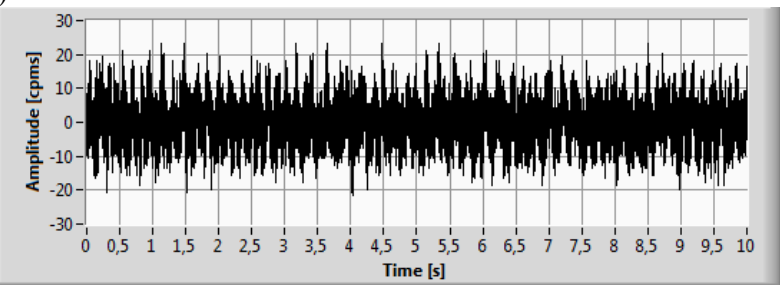

d)

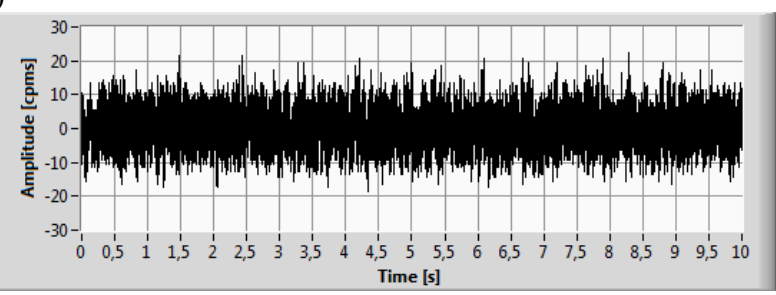

Fig. 4. Time records of signal $x(n)$ (first 10 second) in the experiments: a) LIW5, b) LIW4, c) LIW2, d) LIW1.

\section{Analysis of signals using DWT}

The Discrete Wavelet Transform (DWT) is the most generally used time-frequency filtering method [17]. DWT provides good resolution in both time and frequency domains by reducing additive noises. The DWT method enables a multiresolution analysis by decomposing the discrete signal $x(n)$ into low and highfrequency components. To determine DWT, we can applied an iterative Mallat algorithm [17, 18]. This method uses low-band and high-band filters determined by a mother wavelet function and a corresponding scaling function. A signal $x(n)$ is decomposed into low $a_{j, k}$ (approximations) and high $d_{j, k}$ (details) frequency components (Fig. 5) with multi-level filter banks [19]:

$$
\begin{aligned}
& a_{j, k}=\sum_{l} h(l-2 k) a_{j-1, m} \\
& d_{j, k}=\sum_{l} g(l-2 k) a_{j-1, m}
\end{aligned}
$$

where: $j$ is a parameter that influences on the scaling of the DWT; $k$ is related to the translation within each level of the wavelet function; $l$ is the number of level, and is an integer scale; $h(l)$ and $g(l)$ are low-pass and high-pass quadrature filters respectively; $m$ is used in the scaling function as a translation corresponding to the scaling level $j$. The wavelet transform contains different families 
of wavelet functions, such as Daubechies, Haar, etc. The daubechies $2(\mathrm{db} 2)$ wavelet has been selected for analysis of signals received from the scintillation detector. LabVIEW software [20] was used to analyze of the signals (Fig. 6). Decomposition of the $x(n)$ signal into low- and high-frequency components in the LIW 5 experiment is shown in Figs. 7 and 8.

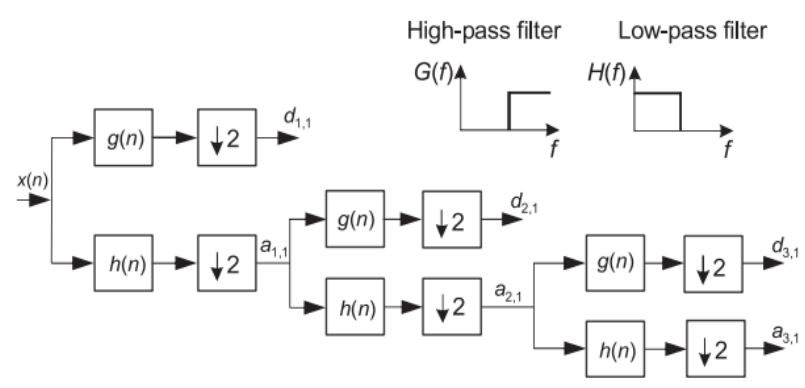

Fig. 5. An example of signal decomposition by the DWT. The operator $\downarrow 2$ means down-sampling by two [19].

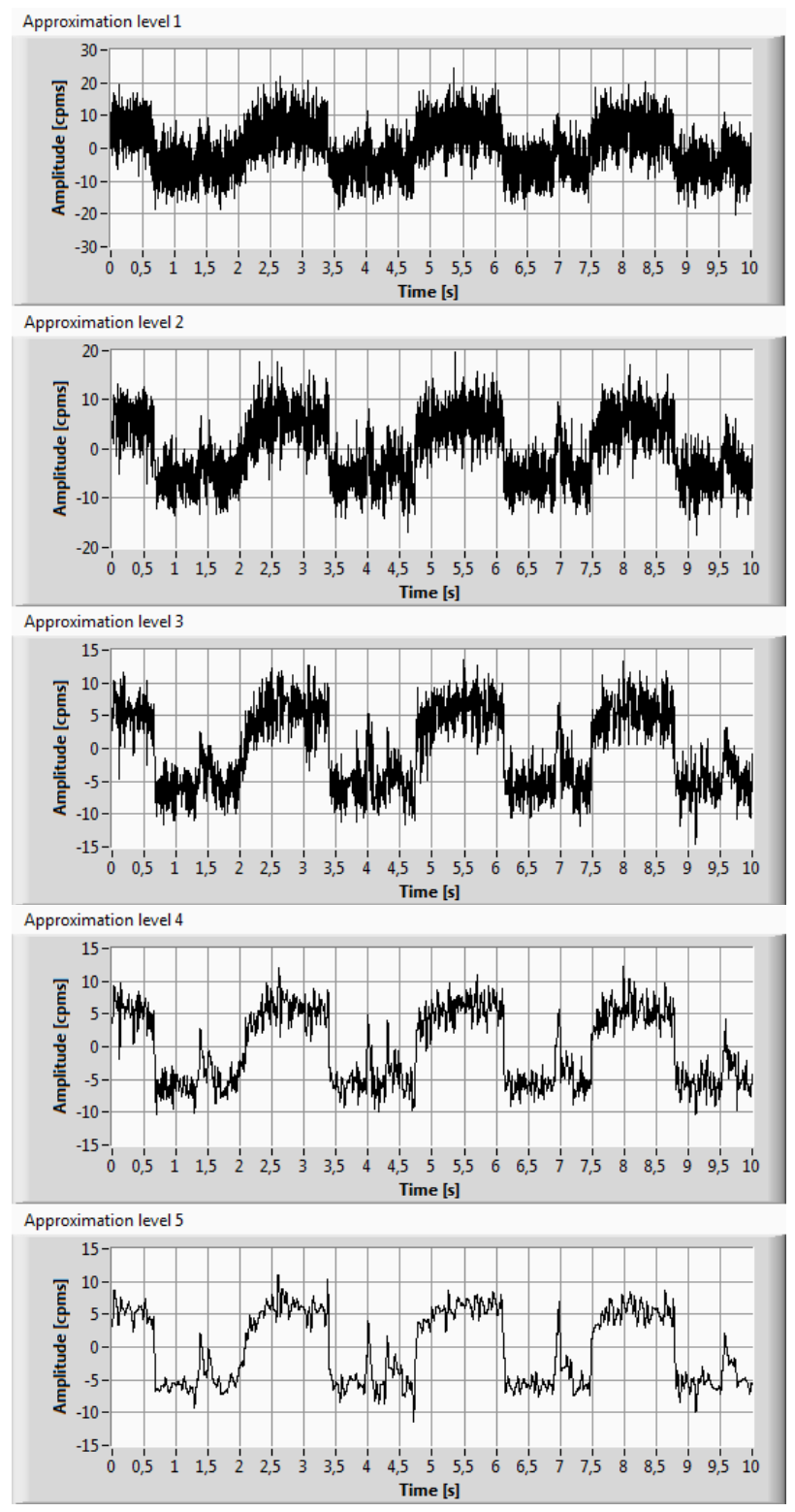

Fig. 7. Decomposition of the $x(n)$ signal into low-frequency components (approximations) in the LIW5 experiment.

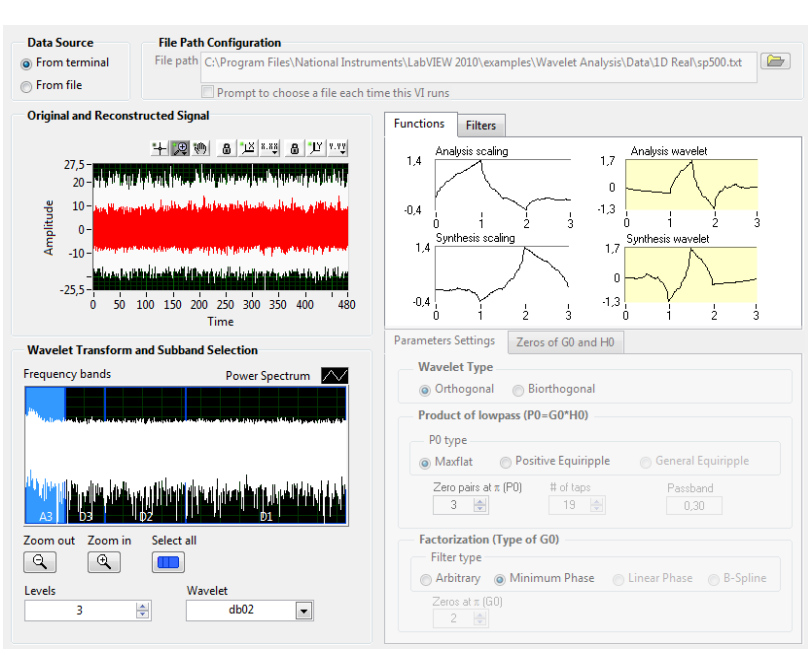

Fig. 6. Configuration window of the Multiresolution Analysis module in the LabVIEW environment.

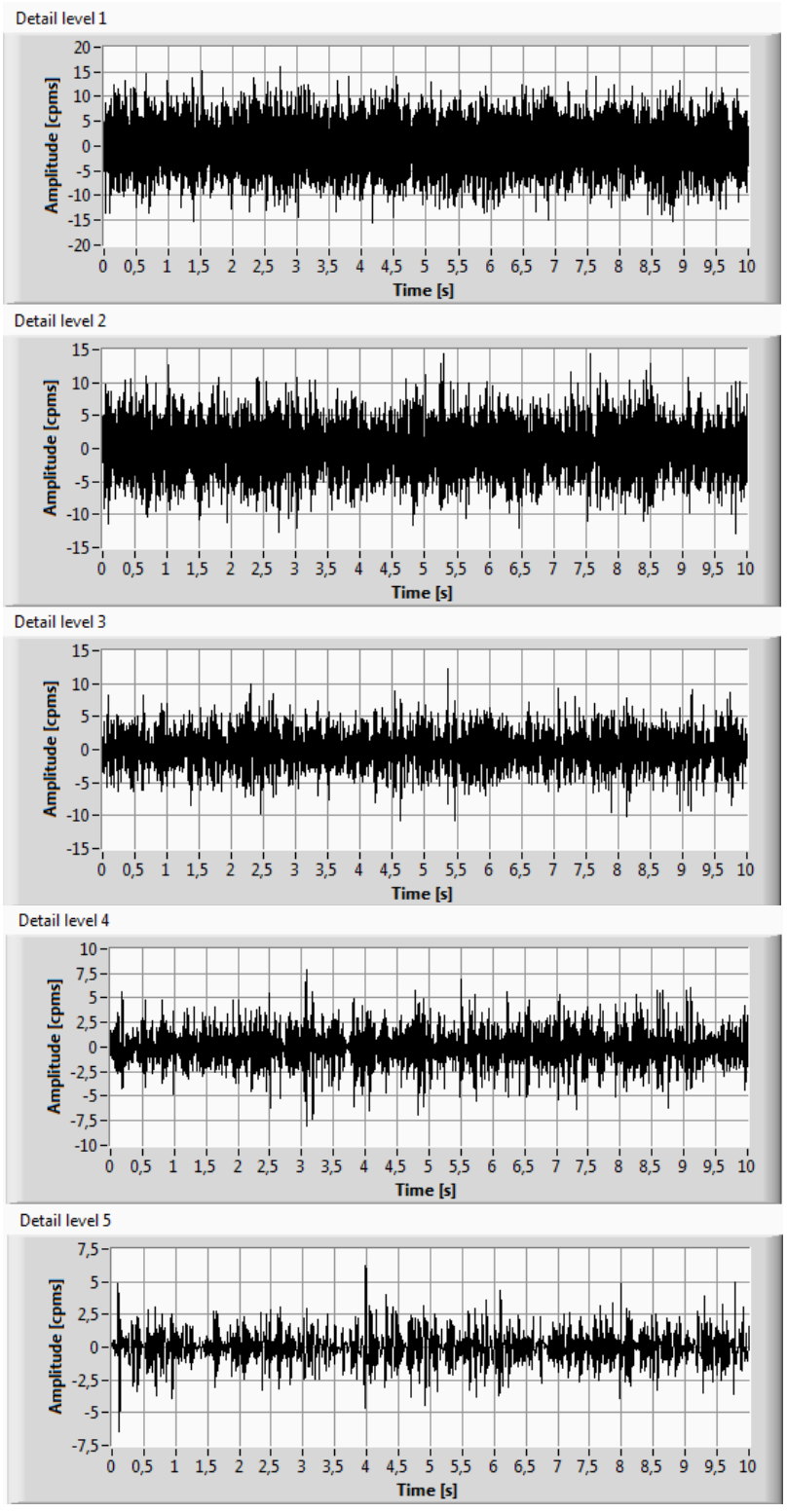

Fig. 8. Decomposition of the $x(n)$ signal into high-frequency components (details) in the LIW5 experiment. 
A similar decomposition was made for the other three types of flows: plug flow (LIW4), transitional plug bubble flow (LIW2), and bubble flow (LIW1). Next, the mean energy of subsequent approximations for 1-5 levels of decomposition was calculated [21].

\section{Results}

Table 1 presents the results of calculations. The mean energy values for the approximations of signal $x(n)$ for five levels of decomposition and for the four analyzed flow regimes are given. In Figure 9, these results are presented graphically.

Table 1. The mean energy values of the approximations for different types of flow (db2 wavelet).

\begin{tabular}{|c|c|c|c|c|}
\hline $\begin{array}{c}\text { Decomposi- } \\
\text { tion level of } \\
\text { signal } x(n) \\
{[-]}\end{array}$ & $\begin{array}{c}\text { LIW 5 } \\
\text { Slug } \\
\text { flow } \\
{\left[\mathrm{cpms}^{2}\right]}\end{array}$ & $\begin{array}{c}\text { LIW 4 } \\
\text { Plug } \\
\text { flow } \\
{\left[\mathrm{cpms}^{2}\right]}\end{array}$ & $\begin{array}{c}\text { LIW 2 } \\
\text { Transitional } \\
\text { plug - } \\
\text { bubble flow } \\
{\left[\mathrm{cpms}^{2}\right]}\end{array}$ & $\begin{array}{c}\text { LIW 1 } \\
\text { Bubble } \\
\text { flow } \\
{\left[\mathrm{cpms}^{2}\right]}\end{array}$ \\
\hline 1 & 41.55 & 28.14 & 24.85 & 17.01 \\
\hline 2 & 33.64 & 20.44 & 17.23 & 9.37 \\
\hline 3 & 29.73 & 16.58 & 13.34 & 5.52 \\
\hline 4 & 27.72 & 14.39 & 11.19 & 3.53 \\
\hline 5 & 26.64 & 12.52 & 9.2 & 2.17 \\
\hline
\end{tabular}

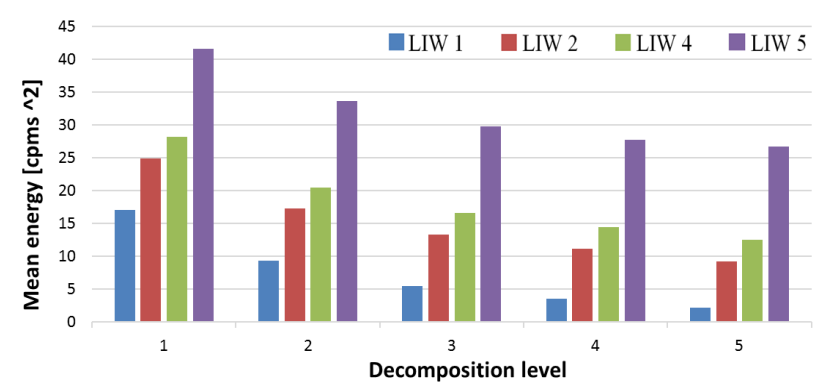

Fig. 9. Comparison of energy of approximations for different levels of decomposition ( $\mathrm{db} 2$ wavelet).

As can be seen from the Table 1 and Fig. 9, the energies of approximations are significantly different for analysed flow regimes. On the other hand, the energy ratios for decomposition at levels from 1 to 5 have similar values.

\section{Conclusion}

In this article, the signals recorded from scintillation detector for four structures of water-air flow as slug, plug, bubble, and transitional plug-bubble flows in the horizontal pipeline were analyzed using the Discrete Wavelet Transform. Using DWT, the signals were decomposed into low (approximations) and high (details) frequency components using db2 type wavelet. This type of wavelet and decomposition level were selected experimentally. Then the mean energy of approximations was calculated at the individual levels of decomposition.
Based on the obtained results it can be stated that the energies of approximations are significantly different for each type of flow and may be useful to the recognition of flow pattern. For the types of flows analyzed, the energy ratios for decomposition levels 1 - 5 have similar values.

In the authors' opinion, the energy of approximation can be used as one of the parameters (along with others in time and frequency domain $[13,14,22-24])$ to recognize the liquid-gas flow regime using artificial intelligence methods.

This work is financed by Polish Ministry of Science and Higher Education under the program "Regional Initiative of Excellence" in 2019 - 2022. Project number 027/RID/2018/19, funding amount 11999900 PLN.

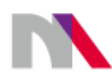

\section{References}

1. Y. Chen, et al, Chem. Eng. Sci. 129,156-167 (2015)

2. S. Blaney, H. Yeung, Flow Meas. Instrum. 19, 57-66 (2008)

3. O. Arjmandi-Tash, A. Zarghami, R. Zarghami, Particul. Sci. Technol. 32, 498-505 (2014)

4. G.H. Roshani, et al, Measurement 129, 296-301 (2018)

5. C.M. Salgado, Prog. Nucl. Energy 52, 555-562 (2010)

6. Z. Sun, H. Zhang, Meas. Sci. Technol. 19, 015401 (2008)

7. G.H. Roshani, et al, Measurement 62, 25-32 (2015)

8. G.H. Roshani, E. Nazemi, Flow Meas. Instrum. 58, 6-11 (2017)

9. M. Khorsandi, et al, Radiat. Meas. 59, 183-187 (2013)

10. H. Ding, et al, Flow Meas. Instrum. 18, 37-46 (2007)

11. T. Elperin, M. Klochko, Exp. Fluids 32, 674-682 (2002)

12. E.S. Rosa, et al, Int. J. Multiphas. Flow 36, 738-754 (2010)

13. R. Hanus, et al., EPJ WoC 114, 02036 (2016)

14. B.M. Abbagoni, H. Yeung, Meas. Sci. Technol. 27, 084002 (2016)

15. R. Hanus, et al., Flow Meas. Instrum. 60, 17-23 (2018)

16. R. Hanus, Int. J. Multiphas. Flow 72, 210-217 (2015)

17. S. Mallat, A wavelet tour of signal processing, (Academic Press, third ed. Burlington 2009)

18. M.L.S. Indrusiak, S.V. Möller, Exp. Therm. Fluid Sci. 35, 319-327 (2011)

19. A.B. Slama, et al, Metrol. Meas. Syst. 25(1), 87-101 (2018)

20. National Instruments LabVIEW, ttp://www.ni.com

21. J. Krupa, BSc thesis, Rzeszów University of Technology, Rzeszów 2018 (in Polish)

22. R. Hanus, et al., EPJ WoC 114, 02035 (2016)

23. R. Hanus, et al., EPJ WoC 143, 02033 (2017)

24. R. Hanus, M. Zych, M. Kusy, P. Hanus, Przegl. Elektrotech. 94(8), 146-149 (2018) (in Polish) 